\title{
Relación entre la flexibilidad lumbar e isquiotibial en pentatletas de la Escuela Militar de Cadetes
}

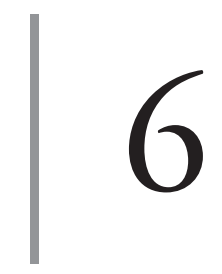

https://doi.org/10.21830/9789585241466.06

Diego Fabricio Rodriguez-Camacho ${ }^{1}$
Juan Felipe Correa-Mesa ${ }^{2}$

\section{Resumen}

Objetivo: estimar la relación entre los resultados de las pruebas Sit and Reach, la prueba de Schober y la prueba pasiva de elevación de la pierna recta (PEPR) en siete pentatletas de la Escuela Militar de Cadetes "General José María Córdova” (ESMIC) de Bogotá, D. C. Colombia. Métodos y materiales: el estudio fue de tipo descriptivo, de asociación y diseño transeccional, que buscó determinar en un momento preciso la relación entre los resultados de la aplicación de la prueba de Sit and Reach, la prueba de Schober y la prueba pasiva de elevación de la pierna recta. (PEPR) en pentatletas de la ESMIC. La muestra incluyó siete participantes, seleccionados a conveniencia. Resultados: en lo que respecta a la flexión lumbar, los sujetos obtuvieron un valor promedio de 4,14 cm, con desviación estándar de 0,87. Para la extensión lumbar se encontró que todos los sujetos se encuentran debajo de los parámetros normales, con un promedio de $1,21 \mathrm{~cm}$ y desviación estándar de 0,58. En la elasticidad isquiotibial se observó que, a excepción de dos sujetos, toda la muestra presenta acortamiento muscular moderado a severo. El promedio de resultado fue similar en las extremidades derecha e izquierda con promedios de $33,1^{\circ}$ y $33,3^{\circ}$, respectivamente. Al aplicar el test de Sit and Reach se halló un resultado promedio de $9,91 \mathrm{~cm}$, valor que está por debajo del rango mínimo aceptado. Conclusiones: sobre la base de la muestra medida en este estudio se

1 Fisioterapeuta, MSc. Fisioterapia del Deporte y la Actividad Física. Grupo de Investigación Rendimiento Físico Militar (Renfimil), Escuela Militar de Cadetes "General José María Córdova”, Bogotá, D.C., Colombia. Contacto: diefrodriguezcam@unal.edu.co - https://orcid.org/0000-0002-7244-8926

2 Fisioterapeuta, MSc. Fisioterapia del Deporte y la Actividad Física. Contacto: juanfcorreames@ unal.edu.co - https://orcid.org/0000-0001-9003-966X 
puede afirmar que no existe una relación entre las diferentes pruebas de flexibilidad, con la excepción de la prueba de Sit and Reach y la flexibilidad de la pierna izquierda, que tienen una correlación negativa. Por lo tanto, la caracterización de la flexibilidad requiere varias medidas complementarias para analizar y justificar las disfunciones en la cadena posterior.

Palabras clave: flexibilidad muscular, Deporte, Rango de movimiento, músculos isquiotibiales.

\section{Introducción}

La flexibilidad es una capacidad física involucrada en la práctica deportiva, y al igual que la fuerza, la potencia y la resistencia, debe entrenarse en espacios planificados y cuantificados con el fin de mejorar el rango de movimiento articular (1). Ahora bien, en la literatura se han reportado distintos beneficios de trabajar la flexibilidad, dentro de los cuales se encuentran cambios térmicos en el tejido conectivo, disminución del dolor, prevención de lesiones e incluso se considera la estrategia predilecta en diversos deportes para la activación y vuelta a la calma. No obstante, actualmente se han debatido estos beneficios debido a la diversidad de posturas en relación con la comprensión del concepto, ya que se han confundido las características extrínsecas e intrínsecas que influyen y las estrategias terapéuticas utilizadas para mejorar el rango de movimiento articular $(1,2)$.

De hecho, en el lenguaje cotidiano se utilizan los conceptos de flexibilidad y estiramientos como sinónimos. Sin embargo, se debe aclarar que la flexibilidad hace referencia al factor intrínseco característico de cada persona que involucra los distintos componentes intra- y extraarticulares (hueso, cartílago, ligamento, músculo, tendón, fascia y piel) y que se traduce en la capacidad de una articulación o conjunto de articulaciones para llevar a cabo un rango de movimiento en un plano y eje específicos (3). En contraste, el estiramiento es un factor extrínseco, modificable y que se utiliza de manera habitual dentro del trabajo de calentamiento y vuelta a la calma en el deporte, así como para prevenir las lesiones y mejorar el rendimiento del atleta (4).

Ahora bien, dentro del tema de flexibilidad se debe abordar el de estiramiento, el cual genera los siguientes efectos agudos: por un lado, los cono- 
cidos como viscoelásticos, los cuales están asociados a los cambios en el rango de movimiento y resistencia al estiramiento debido a cambios en la relajación del estrés, fluencia e histéresis del tejido. Por otro lado, los efectos neurales resultan de la disminución de los índices de excitabilidad de las motoneuronas, que como efecto magno producen relajación y disminución de la capacidad para producir potencia durante la actividad deportiva, aunque se genera un alargamiento temporal del músculo $(4,5)$.

No obstante, como se mencionó, también se encuentran distintas posturas en relación con los beneficios del estiramiento, teniendo en cuenta que las lesiones musculares son citadas como las más frecuentes en la práctica deportiva. Por ejemplo, estudios epidemiológicos han reportado que la disminución de la flexibilidad constituye un factor de riesgo para que se genere una lesión muscular aguda, y por tanto debe ser entrenada como elemento protector (6). Sin embargo, también se han utilizado estudios in vitro para argumentar los cambios en la viscoelasticidad miotendinosa por el incremento excesivo de la temperatura en el músculo previo a la actividad, lo cual disminuye su capacidad para absorber la energía y puede generar una lesión. De esta manera, aunque la información encontrada difiere entre los autores, se requiere precisar la modalidad o técnica de estiramiento aplicada y cuál es su respuesta (6).

En este contexto, la literatura reporta que las variantes del estiramiento más referidas son la estática y dinámica, las cuales generan diferentes efectos. Por ejemplo, en los estiramientos estáticos se afecta principalmente la fase excéntrica del movimiento debido a un cambio en el ciclo de alargamiento-acortamiento, en el cual se disminuye el retroceso elástico y la rigidez de la unión miotendinosa, que es necesaria para almacenar la energía elástica. En consecuencia, el resultado obtenido, sumado a la inhibición de las motoneuronas por depresión del reflejo $\mathrm{H}$, es la disminución de la capacidad de producción de la potencia en el músculo estirado, lo que además favorece el desbalance con el músculo antagonista debido a la inhibición recíproca (5).

Además, autores como Alikhajeh et al. mencionan que la disminución del rendimiento que ocasiona el estiramiento pasivo se debe a que este 
aumenta el compliance de la unidad musculotendinosa, lo cual disminuye su rigidez y por tanto reduce el desarrollo de la fuerza. Adicionalmente, esta respuesta genera inhibición neural y disminución del impulso neural hacia el músculo, lo cual trae como consecuencia la reducción en la producción de potencia (5).

En otros estudios se ha encontrado que el estiramiento estático tiene implicaciones negativas para el desempeño de actividades que exijan potencia, por ejemplo, el salto: en el drop jump se evidencia una disminución en la altura de $4,4 \%$ a 7 \% y en squat jump de 4,3 \% a $4 \%$, así como una disminución de la fuerza de contracción máxima voluntaria del 9,5\%. Sin embargo, dado que no son claros los parámetros ni las técnicas con las cuales se abordó la flexibilidad en estas investigaciones, se puede afirmar que su justificación continúa siendo controversial (7-10).

Por lo contrario, el estiramiento dinámico utilizado previo a la actividad genera aumento de la temperatura central a partir de movimientos que preparan y estimulan la coordinación de patrones de movimiento asociados con el gesto deportivo. Esto incrementa a su vez la sensibilidad de los receptores nerviosos, aumenta la velocidad del impulso nervioso y, por tanto, genera contracciones musculares más rápidas y eficientes, lo cual brinda insumos importantes para que el individuo adopte posturas simples y complejas propias del deporte (5). Estas características hacen que este tipo de estiramiento brinde beneficios preventivos al deportista, considerando que se expone a sufrir lesiones musculoesqueléticas desde el momento en que comienza la práctica deportiva (11).

Ahora bien, teniendo en cuenta que la flexibilidad implica una compleja coordinación entre estructuras de tejido conectivo, y que se comprende como una cualidad física condicional, es necesario entonces que sea estimulada por medio de un proceso planificado y con objetivos puntuales. Así lo argumenta el trabajo de Ayala et al., quienes reportan mejoría en el rango de movimiento de cadera luego de un programa de estiramientos activos durante doce semanas en una frecuencia de tres veces por semana con dosis diarias de 180 segundos en personas con y sin retracción de músculos isquiotibiales 
(12). Sin embargo, se debe tener en cuenta que para identificar el verdadero impacto que tiene entrenar la flexibilidad mediante el estiramiento, es indispensable que en la práctica profesional se utilicen las herramientas apropiadas para medirla y garantizar las estrategias terapéuticas correctivas.

Acorde con lo anterior, dentro de los distintos procesos de caracterización deportiva y valoraciones preparticipación, la medición de la flexibilidad como cualidad física condicional resulta fundamental para identificar factores de riesgo que influyan en el gesto deportivo. Por esta razón, en la práctica clínica se ha incluido frecuentemente la medición de la cadena muscular posterior de manera longitudinal con énfasis en la zona isquiotibial y lumbar baja por medio del test Sit and Reach, el cual se ha convertido en un elemento preferente para los distintos profesionales que interactúan en el deporte debido a su fácil aplicación y su elevada fiabilidad relativa intraexaminador $(0,89-0,99)(13,14)$.

Sin embargo, aunque el test de Sit and Reach tiene validez de criterio media moderada para estimar la flexibilidad isquiotibial ( $\mathrm{rp}=0,46-0,67)$, y media baja para estimar la flexibilidad lumbar ( $r p=0,16-0,35)(15)$, en la práctica profesional se puede generar confusión en relación con las causas por las cuales se obtienen resultados cuantitativos bajos cuando se aplica el test. Esto se debe a que la prueba involucra el componente postural y la estimulación de la cadena muscular posterior de tronco, de manera que una limitación de esta cadena puede arrojar un falso positivo si el examinado tiene dificultad para posicionarse y ejecutar el test, teniendo en cuenta que esta cadena se complementa en miembro inferior a partir de la articulación de cadera (16).

Asimismo, es probable que se deba discriminar objetiva y cuantitativamente cuál es el componente de la cadena que presenta la disfunción, de tal manera que se cuente con insumos para realizar una evaluación más acertada y con el menor sesgo posible, lo cual resulta indispensable para la prescripción de estiramientos que mejoren la flexibilidad en el deportista y de la población en general. Al respecto, en la literatura se reportan distintas pruebas que corroboran los resultados obtenidos en el test de Sit and Reach: 
por ejemplo, se destacan el test de Schober para medir la movilidad en columna lumbar (17) y el test pasivo de elevación de la pierna recta (PEPR) o test 90:90, el cual tiene como objetivo medir la elasticidad isquiotibial partiendo de flexión de rodilla a $90^{\circ}$ hacia la extensión completa (18).

En respuesta a estas consideraciones, el objetivo de la presente investigación tuvo como finalidad estimar la relación entre los resultados de la aplicación del test de Sit and Reach, test de Schober y test pasivo de elevación de la pierna recta (PEPR) en pentatletas de la Escuela Militar de Cadetes “General José María Córdova” (Esmic) de Bogotá, D. C., Colombia.

\section{Metodología}

El estudio fue de tipo descriptivo, de asociación y diseño transeccional, que buscó determinar en un momento específico la relación entre los resultados de la aplicación del test de Sit and Reach, test de Schober y test pasivo de elevación de la pierna recta (PEPR) en pentatletas de la Esmic. La muestra incluyó siete participantes, seleccionados a conveniencia. Como criterios de inclusión se tuvieron en cuenta aquellos sujetos que formaban parte de la selección de pentatlón de la Esmic, que fueran de sexo masculino y que hubieran comprendido y diligenciado el consentimiento informado suministrado por el investigador. En los criterios de exclusión se contemplaron todos aquellos factores que pudieran sesgar los resultados de las pruebas: presentar alguna lesión musculoesquelética en la zona dorsolumbar y en miembros inferiores que generaran dificultad para adoptar la posición y ejecutar las pruebas. Así mismo, la práctica simultánea de otro deporte.

Para medir la flexibilidad de columna lumbar se utilizó el test de Schober de acuerdo con el protocolo establecido en la literatura (17); para medir la elasticidad isquiotibial se aplicó el test pasivo de elevación de la pierna recta (PEPR) siguiendo los criterios desarrollados para su correcta ejecución (18). Para la medición en conjunto a nivel lumbar e isquiotibial se utilizó el test de Sit and Reach clásico siguiendo las consideraciones y requisitos necesarios para su correcta aplicación descritos en la literatura $(12,15)$. 
En cuanto al análisis de los datos, inicialmente se reportaron los resultados descriptivos procesados en el programa $\mathrm{R}$ versión 3.6.0, en el cual se ubicó la información en tablas y se calcularon el promedio y desviación estándar para cada variable. Adicionalmente, para el análisis correlacional de las variables se utilizó el coeficiente de correlación lineal de Pearson, el cual es una prueba estadística que permite identificar el grado de relación entre dos variables medidas en un nivel por intervalos (19).

Las consideraciones éticas se basaron en el artículo 11 de la Resolución N. ${ }^{\circ} 008430$ de 1993 del Ministerio de Salud de Colombia, con base en el cual este estudio se clasifica en la categoría de riesgo mínimo, por cuanto se llevaron a cabo procedimientos rutinarios y comunes que no manipularon la conducta de los sujetos (20). También se contemplaron los principios éticos de la declaración de Helsinki para investigaciones médicas en seres humanos (21).

\section{Resultados}

Durante la aplicación de los test se encontró que, en lo que respecta a la flexión lumbar, los sujetos obtuvieron un valor promedio de 4,14 cm con desviación estándar de 0,87 , lo cual evidencia que la mayoría de la muestra se encuentra dentro de rangos normales, a excepción de un sujeto que solamente logró $2,5 \mathrm{~cm}$ de flexión. Para la extensión lumbar se encontró que todos los sujetos se encuentran debajo de los parámetros normales, con un promedio de 1,21 cm y desviación estándar de 0,58 (tabla 1).

Tabla 1. Resultados de los test Schober, PEPR y Sit and Reach

\begin{tabular}{ccc}
\hline Test utilizado & Promedio & Desviación estándar \\
\hline Schober flexión lumbar & $4,14 \mathrm{~cm}$ & 0,87 \\
Schober extensión lumbar & $1,21 \mathrm{~cm}$ & 0,58 \\
PEPR derecha & $33,1^{\circ}$ & 10,70 \\
PEPR izquierda & $33,3^{\circ}$ & 8,64 \\
Sit and Reach & $9,91 \mathrm{~cm}$ & 5,60 \\
\hline
\end{tabular}

Fuente: Original de los autores. 
Ahora bien, al medir la elasticidad isquiotibial se observó que, a excepción de dos sujetos, toda la muestra presenta acortamiento muscular moderado a severo, teniendo en cuenta que la literatura acepta como valor límite un ángulo de $20^{\circ}$ partiendo de $90^{\circ}$ de flexión de rodilla. El promedio de resultados fue similar en las extremidades derecha e izquierda con promedios de $33,1^{\circ}$ y $33,3^{\circ}$, respectivamente. Sin embargo, el valor de la desviación estándar aporta evidencia de la variabilidad de los resultados entre los sujetos, ya que se encontraron ángulos de hasta $50^{\circ}$ (tabla 1).

Por último, al aplicar el test de Sit and Reach se encontró un resultado promedio de 9,91 cm, valor que está por debajo del rango mínimo aceptado. De acuerdo con este hallazgo y según la clasificación propuesta por el American College of Sports Medicine, todos los sujetos requieren entrenar la flexibilidad. Adicionalmente, se obtuvo una desviación estándar de 5,60 debido a que un sujeto obtuvo un valor de $0 \mathrm{~cm}$ durante la prueba (tabla 1 ).

Adicionalmente, se realizó un análisis de correlación de los datos utilizando el coeficiente de correlación lineal de Pearson, con el fin de medir el grado de relación entre el test de Schober y el test de Sit and Reach, y el test pasivo de elevación de la pierna recta (PEPR) con el test de Sit and Reach. Como resultado se obtuvo que el grado de asociación entre el test de Schober y Sit and Reach es muy débil, lo cual permite inferir que no existe una asociación entre el rango de movimiento de flexo-extensión lumbar medido con cinta métrica y la flexión lumbar medida en conjunto con el grupo isquiotibial. Para la correlación entre Sit and Reach y el test de Schober para flexión el valor p encontrado fue de 0.5552 , lo cual muestra que la hipótesis nula no se puede rechazar y por lo tanto no hay una asociación estadística entre este par de variables.

Asimismo, para observar si la correlación entre Sit and Reach y Schober durante la medición de extensión era significativa, se realizó la misma prueba de hipótesis y se obtuvo un valor p de 0.9655 , es decir, no se puede rechazar la hipótesis de no asociación.

En lo que respecta al análisis de la relación entre los resultados del test de elevación de la pierna recta (PEPR) y el test de Sit and Reach para la 
medición de la elasticidad isquiotibial, se encontró asociación lineal negativa significativa. Esto indica que cuando los sujetos obtienen un resultado mayor en el test de Sit and Reach, menor será el resultado al aplicar el test de elevación de la pierna recta en cuanto a los grados de flexión de la rodilla.

Para determinar si la correlación entre Sit and Reach y la elevación de pierna recta en la extremidad derecha es significativa, se realizó la misma prueba de hipótesis y se obtuvo un valor de $\mathrm{p}=0.1132$, es decir que no se puede concluir asociación entre estas dos mediciones. La prueba de hipótesis para la correlación entre Sit and Reach y la pierna izquierda generó un valor $\mathrm{p}=0.0567$, lo cual es significativo al $10 \%$. Esta correlación negativa indica que cuando sea mayor el resultado de Sit and Reach, se esperaría un menor valor para la variable de la elevación de la pierna izquierda.

\section{Discusión}

Como se expuso anteriormente, la flexibilidad es una cualidad física condicional importante para la adecuada ejecución del movimiento y el desempeño durante la práctica deportiva, de manera que para desarrollarla es necesario implementar estrategias adecuadas según el requerimiento de la persona o atleta, así como es fundamental medirla y evaluarla correctamente $(1,13)$. El objetivo de la presente investigación tuvo como finalidad estimar la relación entre los resultados de la aplicación del test de Sit and Reach, test de Schober y test pasivo de elevación de la pierna recta (PEPR) en pentatletas de la Esmic.

De acuerdo con los resultados obtenidos durante la aplicación del test de Schober se observó que la muestra de pentatletas presenta limitación para el movimiento de extensión de columna lumbar, que puede asociarse a un acortamiento de la cadena muscular posterior de tronco, la cual en condiciones normales permite el posicionamiento y correcto funcionamiento céfalo-caudal respetando las tres leyes del cuerpo a las que hace referencia Busquet: equilibrio, economía y confort (16); además, al considerar el concepto de tensegridad, un acortamiento de esta cadena puede tener efectos directos sobre el funcionamiento de las cadenas musculares a nivel 
de miembro inferior, así como involucrar al grupo isquiotibial que funciona como soporte posterior cuando la cadena posterior de tronco intenta estabilizar la pelvis durante el mantenimiento de la posición bípeda (16). La tensegridad brinda la explicación del porqué un grupo de cadenas muscui lares afecta a otro grupo de cadenas distantes, debido a que el cuerpo es un compartimento cerrado con un conjunto de fuerzas que buscan el equilibrio entre la estructura corporal. La tensegridad se refiere a un sistema de autoequilibrio compuesto por un continuo set de cables que resisten a la fuerza de tensión (músculos, tendones, fascia, ligamentos), los cuales trasmiten información y están sostenidos por un conjunto de struts o barrotes (huesos) (figura 1) (22).

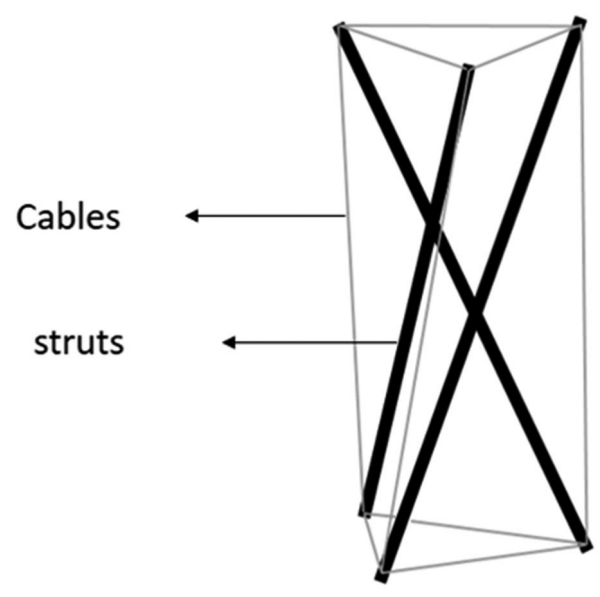

Figura 1. Modelo de tensegridad.

Fuente: Original de los autores.

Sin embargo, a pesar de la continuidad entre la fascia y los músculos que pueden estar anatómicamente distantes entre sí evidenciada en estudios in vitro, existe una escasez de literatura sobre el comportamiento in vivo de estas conexiones (23). Al aplicar la tensegridad a gran escala por fuera de muestras de tejidos en laboratorios, la explicación de la tensegridad en la práctica mostrada en estudios recientes ha brindado evidencia sobre grandes avances en las alteraciones posturales afectadas por orígenes estrucє 
turalmente distantes, inclusive alteraciones en las alineaciones posturales de la espalda baja por lesiones de isquiotibial, como se muestra en un estudio en 34 deportistas de rugby comparados con un grupo control (24).

Por su parte, la prueba de elasticidad isquiotibial mostró que los pentatletas presentan acortamientos de moderados a severos, lo cual evidencia la disfunción mecánica asociada con la cadena de flexión en miembros inferiores. Esto puede desencadenar cambios posturales obligados con el fin de compensar una desventaja mecánica de la cadena antagonista (cadena muscular de extensión), como argumenta $\mathrm{Li}$, con base en indicios de que al estirar los músculos isquiotibiales se puede afectar el movimiento durante la flexión hacia adelante, muestra de la relación agonista-antagonista soportada por la tensegridad en el compartimento del cuerpo humano (25).

Finalmente, los resultados del test de Sit and Reach corroboran los datos obtenidos en las mediciones anteriores, pues demuestran que toda la muestra presenta disminución de la flexibilidad global a nivel lumbar e isquiotibial en conjunto que los hace incapaces de realizar una flexión máxima lumbar con extensión de las rodillas. Estudios recientes han demostrado la relación directa entre el funcionamiento lumbar e isquiotibial: una investigación con treinta sujetos con dolor lumbar clínicamente diagnosticados mostró una disminución significativa en la longitud de los músculos isquiotibiales sin alteraciones de la inclinación pélvica, lo que demuestra una compensación dentro del compartimento corporal por medio por el ajuste de fuerzas sin producir alteraciones posturales significantes (26). De esta manera, al retomar el concepto de tensegridad se puede argumentar que el déficit en el test de Sit and Reach es el resultado de disfunciones miofasciales que en conjunto están generando limitaciones para una adecuada elección, ejecución y mantenimiento de posturas complejas propias de su gesto deportivo, de manera que se ven obligados a desarrollar movimientos compensatorios a expensas del exceso de consumo de energía para cumplir con la demanda deportiva y compensar el equilibrio de tensiones (27).

En síntesis, se ha demostrado que la flexibilidad no solo depende del sistema musculo-esquelético, sino que también depende de los tejidos circun- 
dantes como fascia, tendones y ligamentos, los cuales forman un sistema holístico que en conjunto afectan la postura o el movimiento en el cuerpo humano (el compartimento cerrado integrado por fuerzas de compresión y tensión) (28).

\section{Conclusión}

La flexibilidad como cualidad física condicional es importante para la ejecución del movimiento, la adopción y el mantenimiento de posturas complejas durante la práctica deportiva, por esta razón es necesario medirla con parámetros cuantitativos y válidos que realmente permitan establecer un panorama real de la condición actual del atleta, y de esta manera proponer alternativas terapéuticas para la adquisición y/o desarrollo de parámetros estándar de flexibilidad.

Con base en la muestra medida en este estudio se puede afirmar que no existe una relación entre las diferentes pruebas de flexibilidad, con excepción del test de Sit and Reach y la flexibilidad de la pierna izquierda, los cuales tienen una correlación negativa. Sin embargo, al considerar al cuerpo humano como un sistema de tensegridad, la caracterización de su flexibilidad requiere de mediciones complementarias para analizar y justificar las disfunciones que puedan presentarse para la práctica deportiva.

\section{Agradecimientos}

Esta investigación ha sido realizada gracias a la participación y disposición del grupo de investigación Renfimil de la Escuela Militar de Cadetes "General José María Córdova”. No existe ningún conflicto de interés declarado por parte de los investigadores.

\section{Financiación}

Ninguna declarada por los autores. 


\section{Referencias}

1. Johnson AW, Mitchell UH, Meek K, Feland JB. Hamstring flexibility increases the same with 3 or 9 repetitions of stretching held for a total time of 90 s. Phys. Ther. Sport. 2014 mayo; 15 (2): 101-5. DOI: http://dx.doi.org/10.1016/j.ptsp.2013.03.006

2. Yuktasir B, Kaya F. Investigation into the long-term effects of static and PNF stretching exercises on range of motion and jump performance. J. Bodyw. Mov. Ther. 2009 enero; 13 (1): 11-21. DOI: http://dx.doi.org/10.1016/j.jbmt.2007.10.001

3. Hall C M, Brody LT. Ejercicio terapéutico: recuperación funcional. Barcelona: Paidotribo; 2006.

4. McHugh MP, Cosgrave $\mathrm{CH}$. To stretch or not to stretch: the role of stretching in injury prevention and performance. Scand. J. Med. Sci. Sports. 2009 abril; 20 (2): 169-81. DOI: http://dx.doi.org/10.1111/j.1600-0838.2009.01058.x

5. Alikhajeh Y, Rahimi NM, Fazeli K, Fazeli H. The effect of different warm up stretch protocols on 20m-sprint performance in trained soccer players. Procedia - Soc Behav Sci. 2012; 46: 2210-4.

6. Weldon SM, Hill RH. The efficacy of stretching for prevention of exercise-related injury: a systematic review of the literature. Man. Ther. 2003 agosto; 8 (3):141-50. DOI: http://dx.doi.org/10.1016/s1356-689x(03)00010-9

7. Cronin J, Nash M, Whatman C. The acute effects of hamstring stretching and vibration on dynamic knee joint range of motion and jump performance. Phys Ther. Sport. 2008 mayo; 9 (2): 89-96. DOI: http://dx.doi.org/10.1016/j.ptsp.2008.01.003

8. Nikolaidis PT, Del Coso J, Rosemann T, Knechtle B. Muscle strength and flexibility in male marathon runners: the role of age, running speed and anthropometry. Front. Physiol. 2019 octubre; 10: 1301. DOI: http://dx.doi.org/10.3389/fphys.2019.01301

9. Costa e Silva G, Costa PB, da Conceição RR, Pimenta L, de Almeida RL, Sato MA. Acute effects of different static stretching exercises orders on cardiovascular and autonomic responses. Sci. Rep. 2019 octubre; 9 (1): 15738. DOI: http://dx.doi.org/10.1038/ s41598-019-52055-2

10. Matsuo S, Iwata M, Miyazaki M, Fukaya T, Yamanaka E, Nagata K, et al. Changes in flexibility and force are not different after static versus dynamic stretching. Sports Med. Int. Open. 2019 noviembre; 3 (3): E89-95. DOI: http://dx.doi.org/10.1055/a-1001-1993

11. Rosario JL, Foletto Á. Comparative study of stretching modalities in healthy women: Heating and application time. J. Bodyw. Mov. Ther. 2015 enero; 19 (1): 3-7. DOI: http://dx.doi.org/10.1016/j.jbmt.2013.12.003

12. Ayala F, Sainz de Baranda P, De Ste Croix M, Santonja F. Comparison of active stretching technique in males with normal and limited hamstring flexibility. Phys. Ther. Sport. 2013 mayo; 14 (2): 98-104. DOI: http://dx.doi.org/10.1016/j.ptsp.2012.03.013

13. Miñarro PAL, Andújar PS de B, García PLR, Toro EO. A comparison of the spine posture among several sit-and-reach test protocols. J. Sc.i Med. Sport. 2007 diciembre; 10 (6): 456-62. DOI: http://dx.doi.org/10.1016/j.jsams.2006.10.003 
14. F. Ayala, P. Sainz de Baranda, M. de Ste Croix y F. Santonja. Fiabilidad y validez de las pruebas sit-and-reach: revisión sistemática. Rev. Andal. Med. Deporte. 2012; 5 (2): 57-66.

15. Mayorga-Vega D, Merino-Marban R, Viciana J. Criterion-Related Validity of Sit-andReach Tests for Estimating Hamstring and Lumbar Extensibility: a Meta-Analysis. J Sports Sci Med. 2014; 13 (1): 1-14. Disponible en: https://www.elsevier.es/es-revista-revista-andaluza-medicina-del-deporte-284-articulo-fiabilidad-validez-las-pruebas-si t-and-reach-X1888754612495328

16. Busquet L. Las cadenas musculares. Barcelona: Paidotribo; 2005.

17. Ferraz Pazzinatto M, Valdir Briani R, Bitencourt Oliveira C, Oliveira, Silva D. Testes clínicos para avaliação da coluna lombar e articulação sacroilíaca: revisão de literatura. ConScientiae Saúde. 2014; 13 (4): 650-6. Disponible en: https://www.redalyc.org/ pdf/929/92935317019.pdf

18. Luque Suárez A, Fuente Hervías MT, Barón López FJ, Labajos Manzanares MT. Relación entre el test de elevación de pierna recta y el test ángulo poplíteo en la medición de la extensibilidad isquiosural. Fisioterapia. 2010; 32 (6): 256-63.

19. Hernández Sampieri R, Fernández Collado C, Baptista Lucio P. Metodología de la

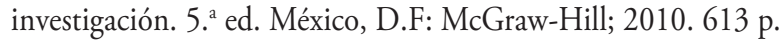

20. Resolución 8493 de 1993 [Internet]. [citado 2 de diciembre de 2019]. Disponible en: https:/www.minsalud.gov.co/sites/rid/Paginas/freesearchresults.aspx?k=RESOLUCION\%2D8430\%2DDE\%2D1993\%2E

21. Brugué JC. Guía investigación clínica 2013: aspectos éticos y jurídicos a tener en cuenta en estudios clínicos en fase II y III. Documenta Universitaria; 2012. 101 p.

22. Cai J, Wang X, Yang R, Feng J. Mechanical behavior of tensegrity structures with Highmode imperfections. Mech. Res. Commun. 2018; 94: 58-63. https://doi.org/10.1016/j. mechrescom.2018.09.006

23. Joshi DG, Balthillaya G, Prabhu A. Effect of remote myofascial release on hamstring flexibility in asymptomatic individuals - A randomized clinical trial. J. Bodyw. Mov. Ther. 2018 julio; 22 (3): 832-7. DOI: http://dx.doi.org/10.1016/j.jbmt.2018.01.008

24. Hennessey L, Watson AW. Flexibility and posture assessment in relation to hamstring injury. Br. J. Sports. Med. 1993 diciembre; 27 (4): 243-6. DOI: http://dx.doi. org/10.1136/bjsm.27.4.243

25. Li Y, McClure PW, Pratt N. The Effect of hamstring muscle stretching on standing posture and on lumbar and hip motions during forward bending. Phys. Ther. 1996 agosto; 76 (8): 836-45. DOI: http://dx.doi.org/10.1093/ptj/76.8.836

26. Fasuyi FO, Fabunmi AA, Adegoke BOA. Hamstring muscle length and pelvic tilt range among individuals with and without low back pain. J. Bodyw. Mov. Ther. 2017 abril; 21 (2): 246-50. DOI: http://dx.doi.org/10.1016/j.jbmt.2016.06.002

27. Carreiro J. Posture, balance and movement. En: An Osteopathic Approach to Children [Internet]. Elsevier; 2009. p. 147-67. Disponible en: https://inkinghub.elsevier.com/ retrieve/pii/B9780443067389000095

28. DellaGrotte J, Ridi R, Landi M, Stephens J. Postural improvement using core integration to lengthen myofascia. J. Bodyw. Mov. Ther. 2008 julio; 12 (3): 231-45. DOI: http://dx.doi.org/ 10.1016/j.jbmt.2008.04.047 